\title{
Analysis of human MutS homolog 2 missense mutations in patients with colorectal cancer
}

\author{
XIAOMEI ZHANG ${ }^{*}$, SENQING CHEN* ${ }^{*}$, JUN YU, YUANYING ZHANG, MIN LV and MING ZHU \\ Department of Molecular Biology, Jiangsu Cancer Hospital, Jiangsu Institute of Cancer Research, \\ Nanjing Medical University Affiliated Cancer Hospital, Nanjing, Jiangsu 210009, P.R. China
}

Received July 28, 2017; Accepted February 2, 2018

DOI: $10.3892 / \mathrm{ol} .2018 .8161$

\begin{abstract}
Germline mutations of DNA mismatch repair gene human MutS homolog 2 ( $h M S H 2)$ are associated with hereditary nonpolyposis colorectal cancer (HNPCC). A total of one-third of these mutations are missense mutations. Several $h M S H 2$ missense mutations have been identified in patients in East Asia, although their function has not been evaluated. In the present study, the role of ten $h M S H 2$ missense mutations in the pathogenesis of colorectal cancer was examined. The hMSH2/hMSH6 protein interaction system was established using yeast two-hybrid screening. Next, the missense mutations were analyzed for their ability to affect the protein interaction of hMSH2 with its partner hMSH6. Additionally, the Sorting Intolerant from Tolerant tool was applied to predict the effects of different amino acid substitutions. The results demonstrated that certain hMSH2 mutations (L173R and C199R) caused a significant functional change in the human hMutS $\alpha$ complex and were identified to be pathological mutations. The Y408C, D603Y, P696L and S703Y mutations partially affected interaction and partly affected the function of hMSH2. The remaining four variants, T8M, I169V, A370T and Q419K, may be non-functional polymorphisms or could affect protein function through other molecular mechanisms. The present study evaluated the functional consequences of previously unknown missense mutations in $h M S H 2$, and may contribute to improved clinical diagnosis and mutation screening of HNPCC.
\end{abstract}

Correspondence to: Dr Ming Zhu, Department of Molecular Biology, Jiangsu Cancer Hospital, Jiangsu Institute of Cancer Research, Nanjing Medical University Affiliated Cancer Hospital, 42 Baiziting Road, Nanjing, Jiangsu 210009, P.R. China E-mail: zm7411@163.com

*Contributed equally

Key words: human MutS homolog 2, missense mutation, yeast two-hybrid, hereditary nonpolyposis colorectal cancer

\section{Introduction}

Hereditary nonpolyposis colorectal cancer (HNPCC) is one of the most common inherited cancer susceptibility syndromes, and is characterized by the early development of a number of types of cancer, including colorectal cancer $(1,2)$. HNPCC is associated with germline mutations in one of the six DNA mismatch repair genes (MMR). These are human MutL homolog 1 ( $h M L H 1), h M S H 2$, human PMS1 homolog 2 ( $h P M S 2)$, human MutS homolog 6 (hMSH6), hPMS1 and $h M L H 3$. The majority of germline mutations in the HNPCC have been identified in $h M S H 2$ and $h M L H 1$ (3). Genetic screening for HNPCC has primarily focused on these two genes and it was identified that half of the germline mutations identified in patients with HNPCC were detected in hMSH2. One-third of the germline mutations are missense mutations (4). The etiology of missense mutations is difficult to be evaluated with respect to nonsense mutations, frameshift mutations and large genomic aberrations. Whether a missense mutation is associated with a disease can be examined by carrier family data analysis or a rigorous case-control study based on a molecular epidemiological survey (5). However, there may be incomplete family data and a difficulty in obtaining epidemiological samples. Evaluation of missense mutations may lack direct experimental functional analysis, thus posing a challenge for the genetic diagnosis of hereditary colorectal cancer. Therefore, assessment of the pathogenicity of missense mutations is required if using the functional assays.

The DNA mismatch repair system aids maintenance of the stability of the genome. The human MSH2 protein is comprised of 934 amino acid residues, and the hMSH6 protein is comprised of 1360 amino acid residues. In vivo, hMSH2 and hMSH6 proteins constitute a heterologous heterodimer (hMutS $\alpha$ ), which binds to the mismatch site and recognizes single base pair mismatches and small insertion or deletion ring structure (6,7). hMLH1 dimerizes with hPMS2 to form a hMutL heterodimer. Next, the hMutS $\alpha$ heterodimer and the hMutL heterodimer constitute a polymer, which recruits other proteins required for mismatch repair (8). The protein-protein interaction of hMSH2 with hMSH6 is the premise of the hMutS $\alpha$ function. Inhibition of the hMSH2-hMSH6 interaction, which is the potential consequence of a missense mutation, may be a major cause of MMR defects in HNPCC (9). 
A previous study established a protein interaction system for $h M L H 1$ and $h P M S 2$ using the yeast two-hybrid system, which was applied for the functional evaluation of missense mutations in $h M L H 1$ genes (10). In the present study, ten missense mutations of $h M S H 2$ that were frequently detected in patients with HNPCC in East Asia were selected. The $h M S H 2$ missense mutations were analyzed for their ability to affect the protein interaction of hMSH2 with its partner hMSH6 in vivo. Additionally, Sorting Intolerant from Tolerant tool (SIFT) tool was applied to predict the effects of amino acid substitution.

\section{Materials and methods}

Strains and plasmids. The wild-type hMSH2 and hMSH6 cDNA were kindly provided by Professor Josef Jiricny (Institute of Molecular Cancer Research, University of Zurich, Zurich, Switzerland). Restriction enzymes and ligase were purchased from Takara Biotechnology Co., Ltd. (Dalian, China). DNA ladders were purchased from GenScript (Piscataway, NJ, USA). The vectors for the yeast two-hybrid system pGADT7 and pGBKT7, the reporter strain of Saccharomyces cerevisiae AH109 and the Escherichia coli strain Top10 were purchased from Clontech Laboratories, Inc. (Mountainview, CA, USA). $E$. coli strain Top10 was used for the genetic engineering of all plasmids.

Characteristics of the variants. A total of 10 missense mutations localized to the entire coding region of $h M S H 2$ gene were analyzed. A frame-shift mutation, c.1664delA, was used as a positive control (11-18). Mutations were identified in patients with HNPCC from East Asia (Table I).

Mutation scanning of normal individuals. A total of 100 healthy Chinese controls, 55 males and 45 females (age range, 35-75 years old), were randomly recruited in The Jiangsu Cancer Hospital (Nanjing, China) from October 2015 to December 2015. Peripheral blood was obtained from normal individuals. Genomic DNA was isolated from $200 \mu$ peripheral blood using the QIAamp DNA Blood Mini kit (Qiagen $\mathrm{GmbH}$, Hilden, Germany), according to the manufacturer's protocol. DNA was subjected to polymerase chain reaction (PCR) amplification of 16 exons of $h M S H 2$ gene using specific primers as listed in Table II, respectively. PCR amplification was performed in a volume of $25 \mu \mathrm{l}$ that contained $50 \mathrm{ng}$ genomic DNA, 0.5 pmol each primer, $2.5 \mu \mathrm{l} 10 \mathrm{X}$ Taq buffer, $2.5 \mu 125 \mathrm{mM} \mathrm{MgCl}{ }_{2}, 2 \mu 12.5 \mathrm{mM}$ dNTPs and $1 \mathrm{U}$ Taq DNA Polymerase (Fermentas; Thermo Fisher Scientific, Inc., Waltham, MA, USA). A thermal cycler (Applied Biosystems Veriti Thermal Cycler; Thermo Fisher Scientific, Inc.) was used for PCR with the following cycling conditions: An initial denaturation step at $95^{\circ} \mathrm{C}$ for 2 min followed by 35 cycles of denaturation at $95^{\circ} \mathrm{C}$ for $0.5 \mathrm{~min}$; annealing at $55^{\circ} \mathrm{C}$ for $0.5 \mathrm{~min}$; and extension at $72^{\circ} \mathrm{C}$ for $1 \mathrm{~min}$, followed by a final extension step at $72^{\circ} \mathrm{C}$ for $5 \mathrm{~min}$. The PCR product was detected on denaturing high-performance liquid chromatography (WAVE System; Transgenomic, Inc., Omaha, NE, USA) to screen the germline mutations of normal individuals of the specific exons. Written informed consent was obtained from all individuals. The study was approved by the Ethics Committee of the Jiangsu Institute of Cancer Research (Nanjing, China).
In silico analysis of hMSH2 variants. The SIFT tool was used to predict whether an amino acid substitution is deleterious $(19,20)$. SIFT prediction is based on a degree of conservation of amino acid residues in sequence alignments derived from closely associated sequences, obtained from PSI-BLAST (http://sift.jcvi.org/). SIFT calculates normalized probabilities for all possible substitutions from the alignment. Positions with normalized probabilities $<0.05$ are predicted to be deleterious, and those $\geq 0.05$ are predicted to be tolerated. In the preset study, 10 missense mutations in the $h M S H 2$ gene were analyzed to identify deleterious variants.

Plasmid construction. Wild-type hMSH2 cDNA was cloned into pGADT7 using the restriction enzymes SfI and XhoI to produce transcriptional AD-fused $h M S H 2$ construct pGADT7-hMSH2. Wild-type $h M S H 6$ cDNA was ligated into pGBKT7 using the restriction enzymes EcoRI and SalI, to produce the transcriptional BD-fused $h M S H 6$ construct pGBKT7-hMSH6. Using pGBKT7-hMSH6 as the template, various domains of hMSH6 were amplified with primers containing EcoRI and SalI restriction sites (Table III). PCR amplification was performed in a volume of $25 \mu \mathrm{l}$ that contained $50 \mathrm{ng}$ pGBKT7-hMSH6, 0.5 pmol each primer, $2.5 \mu \mathrm{l} 10 \mathrm{X}$ Taq buffer, $2.5 \mu 125 \mathrm{mM} \mathrm{MgCl}_{2}, 2 \mu 12.5 \mathrm{mM}$ dNTPs and $1 \mathrm{U}$ TaKaRa LA Taq Polymerase (Takara Biotechnology Co., Ltd.). The Applied Biosystems Veriti Thermal Cycler was used for PCR with the following cycling conditions: An initial denaturation step at $95^{\circ} \mathrm{C}$ for $2 \mathrm{~min}$, followed by 35 cycles of denaturation at $95^{\circ} \mathrm{C}$ for $0.5 \mathrm{~min}$; annealing at $55^{\circ} \mathrm{C}$ for $0.5 \mathrm{~min}$; and extension at $72^{\circ} \mathrm{C}$ for $5 \mathrm{~min}$, followed by a final extension step at $72^{\circ} \mathrm{C}$ for $10 \mathrm{~min}$. The PCR-amplified fragments were then cloned into pGBKT7 using restriction enzymes (EcoRI and SalI), to produce recombinant pGBKT7 plasmids with various hMSH6 domains. Genetic sequencing was then performed using the dideoxynucleotide chain termination method with an automatic sequencer (ABI 3100; Thermo Fisher Scientific, Inc.) (21).

Site-directed mutagenesis and hMSH2 variant construction. The nucleotide substitutions were introduced into the $h M S H 2$ cDNA using overlapping PCR site-directed mutagenesis, as described previously (22). Upstream primers Fa, Ra and downstream primers $\mathrm{Fb}$ and $\mathrm{Rb}$ were synthesized according to the mutation site and PCR reactions were applied. In the first PCR reaction, the upstream fragment was amplified with the upstream primer Fa and the mutant primer Ra, using pGADT7-hMSH2 as template. In the second PCR reaction, the downstream fragment was amplified with the downstream primer $\mathrm{Rb}$ and the mutant primer $\mathrm{Fb}$ using pGADT7-hMSH2 as template. In the third PCR reaction, the final fragment with the target mutant was amplified with primers $\mathrm{Fa}$ and $\mathrm{Rb}$ using the previous two overlapping PCR-amplified products as the template. Specific primers were listed in Table IV. PCR amplification was performed in a volume of $25 \mu \mathrm{l}$ that contained $50 \mathrm{ng}$ template DNA, $0.5 \mathrm{pmol}$ of each primer, $2.5 \mu \mathrm{l}$ 10X Taq buffer, $2.5 \mu \mathrm{l} 25 \mathrm{mM} \mathrm{MgCl}{ }_{2}, 2 \mu 12.5 \mathrm{mM}$ dNTPs and $1 \mathrm{U}$ Taq DNA Polymerase (Fermentas; Thermo Fisher Scientific, Inc.). The Applied Biosystems Veriti Thermal Cycler was used for PCR with the following cycling conditions: An initial denaturation step at $95^{\circ} \mathrm{C}$ for $2 \mathrm{~min}$, 
Table I. Clinical and functional characteristics of the $10 \mathrm{hMSH} 2$ missense variants in the present study.

\begin{tabular}{|c|c|c|c|c|c|}
\hline Exon & $\begin{array}{l}\text { hMSH2 } \\
\text { variation }\end{array}$ & $\begin{array}{l}\text { Nucleotide } \\
\text { substitution }\end{array}$ & Source (Ref.) & $\begin{array}{l}\text { Occurrence } \\
\text { in healthy } \\
\text { individuals }\end{array}$ & SIFT score \\
\hline 1 & $\mathrm{~T} 8 \mathrm{M}$ & c. $23 \mathrm{C}>\mathrm{T}$ & Chinese, Japanese $(11,12)$ & $0 / 100$ & 0.10 \\
\hline 3 & I169V & c. $505 \mathrm{~A}>\mathrm{G}$ & Chinese (13) & $0 / 100$ & 0.30 \\
\hline 3 & L173R & c. $518 \mathrm{~T}>\mathrm{G}$ & Chinese (13) & $0 / 100$ & 0.00 \\
\hline 3 & C199R & c. $595 \mathrm{~T}>\mathrm{C}$ & Chinese (14) & $0 / 100$ & 0.00 \\
\hline 7 & A370T & c. $1108 \mathrm{G}>\mathrm{A}$ & Chinese (15) & $0 / 100$ & 1.00 \\
\hline 7 & Y408C & c. $1223 \mathrm{~A}>\mathrm{G}$ & Chinese $(13,16)$ & $0 / 100$ & 0.00 \\
\hline 7 & Q419K & c. $1255 \mathrm{C}>\mathrm{A}$ & Chinese $(13,16)$ & $0 / 100$ & 0.72 \\
\hline 11 & Frameshift & c.1664delA & Chinese (11) & & \\
\hline 12 & D603Y & c. $1807 \mathrm{G}>\mathrm{T}$ & Chinese (17) & $0 / 100$ & 0.02 \\
\hline 13 & P696L & c. $2087 \mathrm{C}>\mathrm{T}$ & Chinese (15) & $0 / 100$ & 0.00 \\
\hline 13 & S703Y & c. $2108 \mathrm{C}>\mathrm{A}$ & Chinese (18) & $0 / 100$ & 0.01 \\
\hline
\end{tabular}

hMSH2, human MutS homolog 2; SIFT, Sorting Intolerant from Tolerant.

Table II. List of primer sequences used for polymerase chain reaction amplification of the exons of $h M S H 2$ gene.

\begin{tabular}{lll}
\hline hMSH2 gene & \multicolumn{1}{c}{ Forward primer sequence $\left(5^{\prime}-3^{\prime}\right)$} & Reverse primer sequence $\left(5^{\prime}-3^{\prime}\right)$ \\
\hline Exon 1 & GCATTTTCTTCAACCAGGAG & GTCCCTCCCCAGCACGC \\
Exon 2 & GAAGTCCAGCTAATACAGTGC & CTTCACATTTTTATTTTCTACTC \\
Exon 3 & TTTTAAAGTATGTTCAAGAGTTTG & ATCTCCTCTATCACTAGACTC \\
Exon 4 & TTTCATTTTTCTTTTCTTATTCC & TGTAATCACATTTATAATCCATG \\
Exon 5 & CCAGTGGTATAGAAATCTTCG & CCATTCAACATTTTTAACCC \\
Exon 6 & GTTTTCACTAATGAGCTTGCC & GTGGTATAATCATGTGGG \\
Exon 7 & GACTTACGTGCTTAGTTG & TATGAGGACAGCACATTGCC \\
Exon 8 & GATTTGTATTCTGTAAAATGAGATC & CTACAAACTTTCTTAAAGTGGC \\
Exon 9 & TGTCTTTACCCATTATTTATAGG & CAACCTCCAATGACCCATTC \\
Exon 10 & TGGTAGTAGGTATTTATGGAATAC & CATCATGTTAGAGCATTTAGGG \\
Exon 11 & GTACACATTGCTTCTAGTACAC & AGCCAGGTGACATTCAGAAC \\
Exon 12 & ATTCAGTATTCCTGTGTAC & CGTTACCCCCACAAAGC \\
Exon 13 & CGCGATTAATCATCAGTG & GGACAGAGACATACATTTCTATC \\
Exon 14 & GTTACCACATTTTATGTATGG & TTCAAGGGTAGTAAGTTCCC \\
Exon 15 & CTCTTCTCATGCTGTCCC & ATAGAGAAGCTAAGTTAAAC \\
Exon 16 & TAATTACTAATGGGACATTC & TACCTTCATTCCATTACTGG \\
\hline
\end{tabular}

hMSH2, human MutS homolog 2.

followed by 35 cycles of denaturation at $95^{\circ} \mathrm{C}$ for $0.5 \mathrm{~min}$; annealing at $55^{\circ} \mathrm{C}$ for $0.5 \mathrm{~min}$; and extension at $72^{\circ} \mathrm{C}$ for $2 \mathrm{~min}$, followed by a final extension step at $72^{\circ} \mathrm{C}$ for $5 \mathrm{~min}$. The final PCR product was cut with appropriate restriction enzymes (Table IV) and cloned into pGADT7-hMSH2 to replace the wild-type fragment. The recombinant plasmid pGADT7-hMSH2 with the target mutation was verified by sequencing using an automatic sequencer (ABI 3100; Thermo Fisher Scientific, Inc.).

Yeast two-hybrid screening. The Matchmaker GAL4-based two-hybrid system (Clontech Laboratories, Inc.) was used in the present study. The recombinant plasmids pGBKT7-hMSH6 and pGADT7-hMSH2 (wild type or mutant) were co-transformed into S. cerevisiae strain AH109 and plated onto dropout medium SD/-Trp/-Leu (Clontech Laboratories, Inc.) at $30^{\circ} \mathrm{C}$ for $2-4$ days. A total of 10 clones were then selected and inoculated on the dropout medium $\mathrm{SD} /-\mathrm{Trp} /$-leu/-His (Clontech Laboratories, Inc.) at $30^{\circ} \mathrm{C}$ for 2-6 days. The growth status of each transformant in the defect plate was then observed. The clone had normal growth when the transformant grew in two days, whereas slow growth was observed following 2-4 days of culture. No growth was observed $>4$ days of culture. 
Table III. List of primer sequences used for polymerase chain reaction amplification of various hMSH6 domains.

\begin{tabular}{lll}
\hline Domain & \multicolumn{1}{c}{ Forward primer sequence $\left(5^{\prime}-3 '\right)$} & Reverse primer sequence $\left(5^{\prime}-3^{\prime}\right)$ \\
\hline MutS I-IV & CGGAATTCTCTGCCCCTCAAAATTCTG & GCGTCGACTAAGGACCATCACCCCCTCGAC \\
MutS I-V & CGGAATTCTCTGCCCCTCAAAATTCTG & GCGTCGACTATAATTCCTTAATCAAAGTCAG \\
MutS II-IV & CGGAATTCACCAAGGGTACACAGACTTAC & GCGTCGACTAAGGACCATCACCCCCTCGAC \\
MutS II-V & CGGAATTCACCAAGGGTACACAGACTTAC & GCGTCGACTATAATTCCTTAATCAAAGTCAG \\
MutS III-IV & CGGAATTCAGCACTACAAGATCTGGTGC & GCGTCGACTAAGGACCATCACCCCCTCGAC \\
MutS III-V & CGGAATTCAGCACTACAAGATCTGGTGC & GCGTCGACTATAATTCCTTAATCAAAGTCAG
\end{tabular}

\section{Results}

Plasmid construction. Gene engineering was used to construct the recombinant plasmids pGADT7-hMSH2, pGBKT7-hMSH6 from wild-type $h M S H 2$ and $h M S H 6$ cDNA (Fig. 1). Overlapping PCR site-directed mutagenesis was used to construct 11 mutated pGADT7-hMSH2 plasmids. All wild-type and variant plasmids were constructed successfully and verified by sequencing. The hMSH6 protein is comprised of 1,360 amino-acid residues, which can be divided into a N-terminal domain (residues, 1-409), and MutS I, II, III, IV and $\mathrm{V}$ functional domains (MutS $\mathrm{I}$ is the mismatch-binding domain, MutS II is the connector domain, MutS III is the core domain, MutS IV is the clamp domain and MutS V is the ATPase domain) $(6,7)$. These domains all interact with the corresponding functional domains of hMSH2 protein to complete the mismatch repair function in vivo (8) hMSH6 has a large molecular weight which may prevent proper protein folding in yeast. A total of six recombinant pGBKT7 plasmids with various hMSH6 domains were constructed, including pGBKT7-hMSH6-MutS I-IV (residues, 341-1,073), MutS I-V (residues, 341-1,360), MutS II-IV (residues, 518-1,073), MutS II-V (residues, 518-1,360), MutS III-IV (residues, 718-1,073) and MutS III-V (residues, 718-1,360) (Fig. 2) (6,7). All of the pGBKT7 plasmids with different hMSH6 domains were constructed successfully and verified by sequencing.

Mutation identification in 100 normal individuals. A total of 100 individuals were recruited in The Jiangsu Cancer Hospital. Denaturing high-performance liquid chromatography analysis was used to screen the germline mutations of the normal individuals by PCR of the specific exons. None of the missense mutations examined in the present study were identified in the normal controls (Table I).

Prediction of hMSH2 protein activity and structure. The SIFT score of $h M S H 2$ mutations demonstrated that six of them were considered to be intolerable (L173R, C199R, Y408C, D603Y, P696L and S703Y), indicating that these amino acid substitutions may affect the function of the protein. The remaining four mutations (T8M, I169V, A370T and Q419K) were considered to be tolerable (Table I).

Yeast two-hybrid assay of hMSH2 and hMSH6 domains. Recombinant plasmids pGADT7-hMSH2 (wild type) and pGBKT7 with various hMSH6 domains were co-transformed into S.cerevisiae strain $A H 109$ and selected on nutritional defect

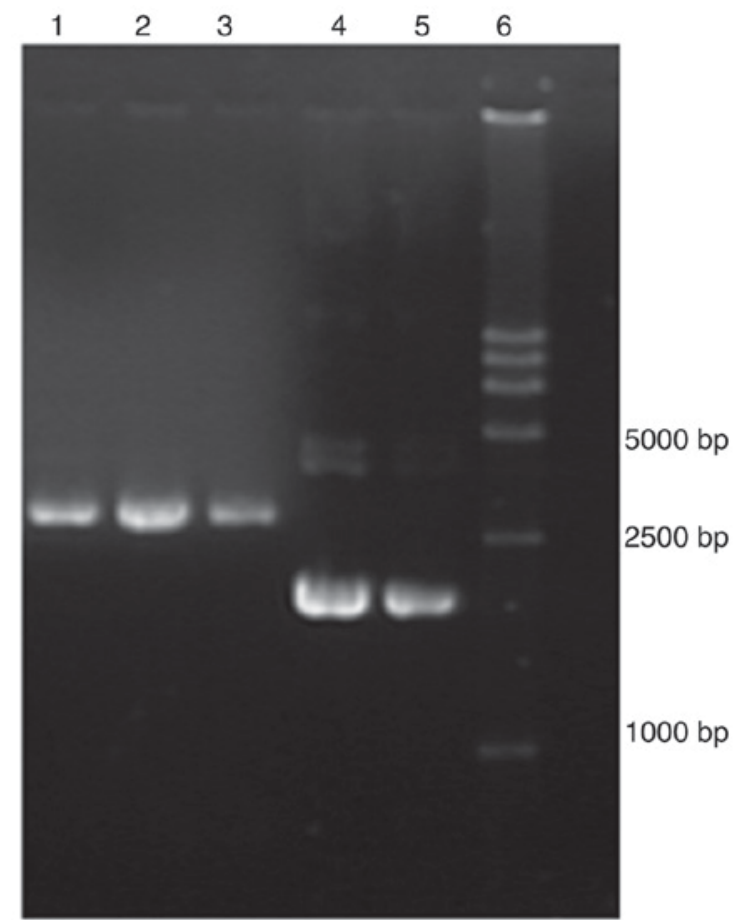

Figure 1. Polymerase chain reaction electrophoresis of plasmid pGBKT7-hMSH6. Lanes 1, 2 and 3, MutS I-V fragments (3.1 kb); lanes 4 and 5, MutS III-V fragments $(1.9 \mathrm{~kb})$; lane 6, DNA ladder.

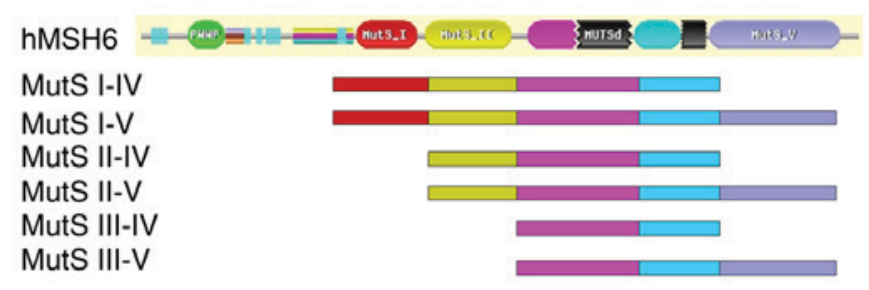

Figure 2. Scope of six hMSH6 domains. hMSH6, human MutS homolog 6. hMSH6 (residues 1-1360), MutS I-IV (residues 341-1073), MutS I-V (residues 341-1360), MutS II-IV (residues 518-1073), MutS II-V (residues 518-1360), MutS III-IV (residues 718-1073) and MutS III-V (residues 718-1360).

plates. The growth status of each transformant was observed (Table V). The negative control was pGADT7/pGBKT7 and the positive control was pGADT7-hMLH1/pGBKT7-hPMS2 (10). It was demonstrated that the AH109 two-hybrid transformant pGADT7-hMSH2/pGBKT7-hMSH6-MutS II-V and pGADT7-hMSH2/pGBKT7-hMSH6-MutS III-V grew on the histidine-deficient medium (SD/-Trp/-leu/-His). The results 
Table IV. List of primer sequences used for polymerase chain reaction amplification of $h M S H 2$ variants and appropriate restriction enzymes for cloning.

\begin{tabular}{|c|c|c|}
\hline Mutation site & Primer sequence (5'-3') & Restriction enzymes \\
\hline c. $23 \mathrm{C}>\mathrm{T}$, & Fa: CTAACGTTCATGATAACTTCATG & AclI \\
\hline \multirow[t]{3}{*}{$\mathrm{T} 8 \mathrm{M}$} & Ra: CTCCAACTGCAGCaTCTCCTTCGGC & HindIII \\
\hline & Fb: GCCGAAGGAGAtGCTGCAGTTGGAG & \\
\hline & Rb: AATTCTGCATCTTCTACAAAAGC & \\
\hline c. $505 \mathrm{~A}>\mathrm{G}$ & Fa: CGGGCCATGGAGGCCCCTGGCAATCTCTCTCAGTTTG & $S f i \mathrm{I}$ \\
\hline \multirow{3}{*}{$\mathrm{I} 169 \mathrm{~V}$} & Ra: GTTTCCTCTGTAcGGAATCCACATAC & HindIII \\
\hline & Fb: GTATGTGGATTCCgTACAGAGGAAAC & \\
\hline & Rb: AATTCTGCATCTTCTACAAAAGC & \\
\hline c. $518 \mathrm{~T}>\mathrm{G}$ & Fa: CGGGCCATGGAGGCCCCTGGCAATCTCTCTCAGTTTG & SfiI \\
\hline \multirow[t]{3}{*}{ L173R } & Ra: CACACAGTCCTcGTTTCCTCTGTATG & HindIII \\
\hline & Fb: CATACAGAGGAAACgAGGACTGTGTG & \\
\hline & Rb: AATTCTGCATCTTCTACAAAAGC & \\
\hline c.595T>C, & Fa: CGGGCCATGGAGGCCCCTGGCAATCTCTCTCAGTTTG & SfiI \\
\hline \multirow[t]{3}{*}{ C199R } & Ra: CGGGTAAAACACgTTCCTTTGGTCC & HindIII \\
\hline & Fb: GGACCAAAGGAAcGTGTTTTACCCG & \\
\hline & Rb: AATTCTGCATCTTCTACAAAAGC & \\
\hline c. $1108 \mathrm{G}>\mathrm{A}$, & Fa: CGGGCCATGGAGGCCCCTGGCAATCTCTCTCAGTTTG & SfiI \\
\hline \multirow{3}{*}{ A370T } & Ra: GCCTCAATTCTGtATCTTCTACAAAAG & XhoI \\
\hline & Fb: CTTTTGTAGAAGATaCAGAATTGAGGC & \\
\hline & Rb: GCCTCGAGTCACGTAGTAACTTTTATTCGTG & \\
\hline \multirow{4}{*}{$\begin{array}{l}\text { c. } 1223 \mathrm{~A}>\mathrm{G} \\
\mathrm{Y} 408 \mathrm{C}\end{array}$} & Fa: GAGAGATTGAATTTAGTGGAAGC & Hind III \\
\hline & Ra: GATTTATACCCTGAcAGAGTCGGTAAC & XhoI \\
\hline & Fb: GTTACCGACTCTgTCAGGGTATAAATC & \\
\hline & Rb: GCCTCGAGTCACGTAGTAACTTTTATTCGTG & \\
\hline \multirow{4}{*}{$\begin{array}{l}\mathrm{c} .1255 \mathrm{C}>\mathrm{A}, \\
\mathrm{Q} 419 \mathrm{~K}\end{array}$} & Fa: GAGAGATTGAATTTAGTGGAAGC & Hind III \\
\hline & Ra: GTTTTTCCAGAGCCTtTATAACATTAGG & XhoI \\
\hline & Fb: CCTAATGTTATAaAGGCTCTGGAAAAAC & \\
\hline & Rb: GCCTCGAGTCACGTAGTAACTTTTATTCGTG & \\
\hline \multirow{4}{*}{$\begin{array}{l}\text { c.1664delA, } \\
\text { frameshift }\end{array}$} & Fa: GAGAGATTGAATTTAGTGGAAGC & HindIII \\
\hline & Ra: CATTTAAAGAAGTCAATTGCTGTTGGTAAATTTAAC & XhoI \\
\hline & Fb: GTTAAATTTACCAACAGCAATTGACTTCTTTAAATG & \\
\hline & Rb: GCCTCGAGTCACGTAGTAACTTTTATTCGTG & \\
\hline c. $1807 \mathrm{G}>\mathrm{T}$, & Fa: GAGAGATTGAATTTAGTGGAAGC & HindIII \\
\hline \multirow[t]{3}{*}{ D603Y } & Ra: GACAACAGCATaTAGCTGAGCTAAC & XhoI \\
\hline & Fb: GTTAGCTCAGCTAtATGCTGTTGTC & \\
\hline & Rb: GCCTCGAGTCACGTAGTAACTTTTATTCGTG & \\
\hline c. $2087 \mathrm{C}>\mathrm{T}$, & Fa: GAGAGATTGAATTTAGTGGAAGC & Hind III \\
\hline \multirow[t]{3}{*}{$\mathrm{P} 696 \mathrm{~L}$} & Ra: GCTGACTCACATaGCACAAAACACC & XhoI \\
\hline & Fb: GGTGTTTTGTGCtATGTGAGTCAGC & \\
\hline & Rb: GCCTCGAGTCACGTAGTAACTTTTATTCGTG & \\
\hline c. $2108 \mathrm{C}>\mathrm{A}$, & Fa: GAGAGATTGAATTTAGTGGAAGC & HindIII \\
\hline \multirow[t]{3}{*}{ S703Y } & Ra: CAGTCCACAATGtACACTTCTGCTG & XhoI \\
\hline & Fb: CAGCAGAAGTGTaCATTGTGGACTG & \\
\hline & Rb: GCCTCGAGTCACGTAGTAACTTTTATTCGTG & \\
\hline
\end{tabular}

$\mathrm{Fa}$, forward primer sequence $\mathrm{a} ; \mathrm{Fb}$, forward primer sequence $\mathrm{b}$; Ra, reverse primer sequence $\mathrm{a}$; Rb, reverse primer sequence $\mathrm{b}$.

demonstrated that hMSH6-MutS II-V and MutS III-V may interact with hMSH2 in the yeast strain AH109. Additionally, the two-hybrid transformants pGADT7/pGBKT7-hMSH6MutS II-V and pGADT7/pGBKT7-hMSH6-MutS III-V did not 

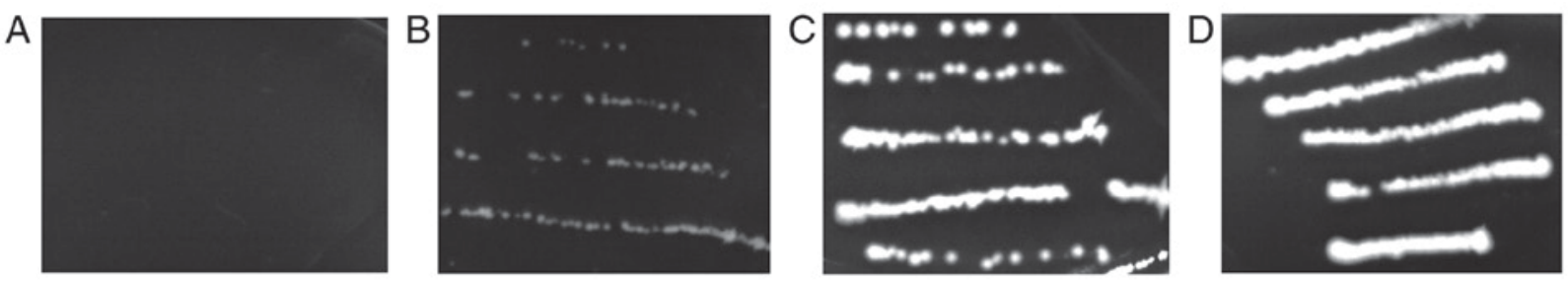

Figure 3. Yeast two-hybrid system for hMSH2 variants and hMSH6-MutS II-V domains. The two-hybrid transformant clones (A) C199R, (B) P696L (C) T8M and (D) wild-type hMSH2 were selected on SD/-Trp/-leu/-His plate, followed by incubation at $30^{\circ} \mathrm{C}$ for 4 days. hMSH2, human MutS homolog 2; SD, synthetic defined.

Table V. Yeast two-hybrid assay of hMSH2 and various hMSH6 domains.

Transformant Growth status

pGADT7/pGBKT7 (negative control)

pGADT7-hMSH2/pGBKT7-hMSH6

$\begin{array}{ll}- & \text { Wild-type } h M S H 2 \\ - & \text { T8M } \\ - & \text { I169V } \\ & \text { L173R } \\ - & \text { C199R } \\ & \text { A370T } \\ - & \text { Y408C } \\ & \text { Q419K } \\ + & \text { c.1664delA } \\ & \text { D603Y } \\ - & \text { P696L } \\ & \text { S703Y }\end{array}$

pGADT7-hMSH2/pGBKT7-hMSH6-MutS

I-IV

pGADT7-hMSH2/pGBKT7-hMSH6-MutS

$\mathrm{I}-\mathrm{V}$

pGADT7-hMSH2/pGBKT7-hMSH6-MutS

II-IV

pGADT7-hMSH2/pGBKT7-hMSH6-MutS

II-V

pGADT7-hMSH2/pGBKT7-hMSH6-MutS

III-IV

pGADT7-hMSH2/pGBKT7-hMSH6-MutS

III-V

pGADT7-hMLH1/pGBKT7-hPMS2

(positive control)

pGADT7/pGBKT7-hMSH6-MutS II-V

pGADT7/pGBKT7-hMSH6-MutS III-V

hMSH2, human MutS homolog 2; +, two-hybrid transformant grew normally in histidine-deficient medium; -, two-hybrid transformant did not grow in the histidine-deficient medium.

grow on the histidine-deficient medium. These results precluded the self-activation of pGADT7/pGBKT7-hMSH6-MutS II-V and pGADT7/pGBKT7-hMSH6-MutS III-V for His reporter gene in yeast strain $A H 109$.

Yeast two-hybrid assay of hMSH2 variants and hMSH6-MutS II-V. Mutant pGADT7-hMSH2 and pGBKT7-hMSH6-MutS II-V plasmids were co-transformed into $S$. cerevisiae strain AH109 and selected on a SD/-Leu-Trp plate. The selected clones were then inoculated on a SD/-Trp/-leu/-His plate. The growth status of each transformant was observed (Table VI). The negative control was pGADT7/pGBKT7 and positive control was pGDAT7-hMSH2/pGBKT7-hMSH6-MutS II-V. It was demonstrated that the yeast two-hybrid transformants T8M, I169V, A370T and Q419K grew normally, the transformants Y408C, D603Y, P696L and S703Y grew slowly, whereas the
Table VI. Yeast two-hybrid Assay of hMSH2 variants and hMSH6-MutS II-V.

hMSH2 variation

Growth status

hMSH2, human MutS homolog 2; +, two-hybrid transformant grew normally in histidine-deficient medium; -, two-hybrid transformant did not grow in the histidine-deficient medium; +/-, two-hybrid transformant had slow growth on the histidine-deficient medium.

transformants L173R, C199R and c.1664delA did not grow on the histidine-deficient medium in yeast strain AH109 compared with wild-type hMSH2 (Fig. 3). The Y408C, D603Y, P696L and S703Y mutations may partly affect the function of hMSH2 in yeast strain AH109. c.1664delA is a frameshift mutation, thus $h M S H 2$ c.1664delA can not bind hMSH6-MutS II-V. The yeast two-hybrid transformant pGDAT7-hMSH2-c.1664delA/pGBKT7-hMSH6-MutS II-V did not grow in the histidine-deficient medium in yeast strain AH109. The L173R and C199R mutants gave similar results to those of c.1664delA, indicating that the mutations prevented the interaction between hMSH2 and hMSH6.

\section{Discussion}

In the present study, 10 missense mutations to $h M S H 2$ were examined in patients with HNPCC from East Asia. The functional consequences of the variants were analyzed based on their genetic, epidemiological and functional characteristics.

In vitro measurement of MMR function has been used as a direct method to evaluate the variants in the $h M S H 2$ gene (23). It was difficult to perform this complex experiment since it required expression and purification of proteins. The 
yeast two-hybrid system has been used as a method to study the interaction between proteins (24). A previous study established a protein interaction system for hMLH1 and hPMS2 using the yeast two-hybrid system, which was applied for the functional evaluation of missense mutations in $h M L H 1$ genes (10). The yeast two-hybrid system has advantages for detecting protein binding: Proteins may maintain a natural conformation to mimic the physiological state in vivo, and this system eliminates the time-consuming steps necessary for protein purification in vitro. A previous study used a similar system to evaluate the partial $h M S H 2$ missense mutations I169V, L173R, Y408C, Q419K and S703Y (25). In the present study, the yeast two-hybrid experimental method has been improved and the results have been evaluated using SIFT to evaluate the enlarged $h M S H 2$ missense mutations. The yeast two-hybrid system was used to analyze the ability of $h M S H 2$ missense mutations to affect the interaction of hMSH2 with its partner hMSH6 in vivo. The growth status of each transformant provided an indication of the protein interaction strength, as the enzyme activity of reporter genes was associated with the degree of protein binding.

Genetic engineering was used to construct recombinant plasmids pGADT7-hMSH2 and pGBKT7-hMSH6. Next, the recombinant plasmids pGADT7-hMSH2 and pGBKT7-hMSH6 were co-transformed into $S$. cerevisiae strain AH109. The results demonstrated that the yeast two-hybrid transformant couldn't grow in histidine-deficient medium, indicating that the interaction between hMSH2 and hMSH6 may be inhibited in yeast $A H 109$. The possible reason behind this may be the inappropriate folding of hMSH6 protein when expressed in yeast strain AH109, owing to its size. Additionally, six recombinant pGBKT7 plasmids with various hMSH6 domains were constructed. The results demonstrated that the transformant pGADT7-hMSH2/pGBKT7-hMSH6-MutS II-V and pGADT7-hMSH2/pGBKT7-hMSH6-MutS III-V were able to grow on the histidine-deficient medium (Table V). These results indicate that hMSH6-MutS II-V and MutS III-V may interact with hMSH2 in yeast strain AH109. The hMSH6 MutS II-V domain contains amino acid residues 518-1,360 of the hMSH6 protein, which comprises the core regions of the full protein. The yeast two-hybrid transformant pGADT7-hMSH2/pGBKT7-hMSH6-MutS II-V was used to construct the interaction system of hMSH2-hMSH6 and to evaluate $h M S H 2$ missense variants (Table VI).

SIFT scores revealed that T8M, I169V, A370T and Q419K were tolerant substitutions. The results of the present study indicated that these variants may be nonfunctional polymorphisms or may affect the protein function through other molecular mechanisms.

SIFT scores revealed that Y408C, D603Y, P696L and S703Y were intolerable substitutions. In the Y408C mutant, tyrosine and cysteine belong to neutral amino acids, but their stereochemistry differs. For the D603Y mutant, aspartic acid is an acidic amino acid, whereas tyrosine is an aromatic amino acid, meaning their reactivities may differ. P696L was associated with a loss of hMSH2 protein expression and high level of microsatellite instability in tumors (15). For S703Y, homology modeling revealed that steric hindrance was increased because of the serine-to-tyrosine mutation at residue 703. Finally, the hydrogen bonds of Ala700 and Glu706 were not formed, affecting the whole molecular structure and leading to inactivation of hMSH2 function (18). The yeast two-hybrid transformant of the four variants grew slowly on the histidine-deficient medium. Therefore, these variants may affect the partial interaction and partly affect the function of hMSH2.

L173R and C199R are highly conserved from yeast to humans (6). SIFT scores revealed that they were intolerable substitutions. For the L173R mutation, leucine is an uncharged hydrophobic amino acid, whereas arginine is a positively charged, weak-basic amino acid. Their functions may differ as a result of differences in solubility and ionization properties. For the C199R mutation, cysteine is an uncharged hydrophilic amino acid, whereas arginine is a positively charged, weak-basic amino acid. Their functions may also differ as a result of differences in stereochemistry and ionization properties. The yeast two-hybrid transformant of the two variants, including c.1664delA, did not grow on histidine-deficient medium. c.1664delA is a frameshift mutation, thus hMSH2 c.1664delA is not able to bind hMSH6-MutS II-V. L173R and C199R may have a similar effect as c.1664delA. Therefore, it was considered that they these mutations may be pathological.

In the present study, segregation studies were not conducted because of the unavailability of family samples. Therefore, the synergistic effect of low-risk alleles of MMR genes, which was previously described (26), was not evaluated in the present study.

In conclusion, the present study established the preliminary hMSH2/hMSH6 protein-interaction system using a yeast two-hybrid system. Combined with SIFT analysis and amino acid analysis, an evaluation system for missense mutations in $h M S H 2$ was established. Using this system, 10 missense mutations in $h M S H 2$ were examined. The results demonstrated that the $h M S H 2$ mutations L173R and C199R caused a functional change in the hMutS $\alpha$ complex and were identified as pathological mutations. The Y408C, D603Y, P696L and S703Y variants partially affected interaction, and may partly affect the function of hMSH2. The remaining four variants, T8M, I169V, A370T and Q419K, may be non-functional polymorphisms or may affect the protein function through other molecular mechanisms. The present study evaluated the functional consequences of several previously unknown missense mutations in the $h M S H 2$ gene, which could contribute to clinical diagnosis and mutation screening of HNPCC.

\section{Acknowledgements}

The authors thank Professor Josef Jiricny (Institute of Molecular Cancer Research, University of Zurich, Switzerland) for providing the hMSH2 and hMSH6 cDNAs.

\section{Funding}

The present study was supported by the Project of Jiangsu Provincial Commission of Health and Family Planning (grant nos. H201510 and H2017035), Jiangsu Provincial Medical Youth Talent (grant no. QNRC2016652), the Natural Science Foundation of Jiangsu Province (grant nos. BK20141490 and BK20161598) and the Research Project of Jiangsu Cancer Hospital (grant no. ZM201107). 


\section{Availability of data and materials}

The datasets used and analyzed during the current study are available from the corresponding author on reasonable request.

\section{Authors' contributions}

MZ designed the study, performed the research and wrote the manuscript. XZ and SC constructed all the plasmids. JY analyzed the data. YZ performed the yeast two-hybrid assay. ML collected the blood samples and performed mutation scanning of normal individuals. All authors read and approved the final manuscript.

\section{Ethics approval and consent to participate}

This research was approved by the Ethics Committee of the Jiangsu Institute of Cancer Research (Nanjing, China) and written informed consent was obtained from all individuals.

\section{Consent for publication}

Informed consent for the publication of any associated data and accompanying images was obtained from all the normal individuals.

\section{Competing interests}

The authors declare that they have no competing interests.

\section{References}

1. Brosens LA, Offerhaus GJ and Giardiello FM: Hereditary colorectal cancer: Genetics and screening. Surg Clin North Am 95: 1067-1080, 2015.

2. Lynch HT and de la Chapelle A: Genetic susceptibility to non-polyposis colorectal cancer. J Med Genet 36: 801-818, 1999

3. InSiGHT: InSiGHT variant databases. https://www.insight-group. org/variants/databases/. Accessed June 29, 2017.

4. Woods MO, Williams P, Careen A, Edwards L, Bartlett S, McLaughlin JR and Younghusband HB: A new variant database for mismatch repair genes associated with Lynch syndrome. Hum Mutat 28: 669-673, 2007.

5. Peltomaki $\mathrm{P}$ and Vasen HF: Mutations predisposing to hereditary nonpolyposis colorectal cancer: Database and results of a collaborative study. The International Collaborative Group on Hereditary Nonpolyposis Colorectal Cancer. Gastroenterology 113: 1146-1158, 1997.

6. Obmolova G, Ban C, Hsieh P and Yang W: Crystal structures of mismatch repair protein MutS and its complex with a substrate DNA. Nature 407: 703-710, 2000

7. Lamers MH, Perrakis A, Enzlin JH, Winterwerp HH, de Wind N and Sixma TK: The crystal structure of DNA mismatch repair protein MutS binding to a GxT mismatch. Nature 407: 711-717, 2000.

8. Jiricny J: The multifaceted mismatch-repair system. Nat Rev Mol Cell Biol 7: 335-346, 2006.

9. Geng H, Sakato M, DeRocco V, Yamane K, Du C, Erie DA, Hingorani $\mathrm{M}$ and Hsieh P: Biochemical analysis of the human mismatch repair proteins hMutSa MSH2(G674A)-MSH6 and MSH2-MSH6(T1219D). J Biol Chem 287: 9777-9791, 2012.

10. Fan Y, Wang W, Zhu M, Zhou J, Peng J, Xu L, Hua Z, Gao X and Wang Y: Analysis of hMLH1 missense mutations in East Asian patients with suspected hereditary nonpolyposis colorectal cancer. Clin Cancer Res 13: 7515-7521, 2007.
11. Wang XL, Yuan Y, Zhang SZ, Cai SR, Huang YQ, Jiang Q and Zheng S: Clinical and genetic characteristics of Chinese hereditary nonpolyposis colorectal cancer families. World J Gastroenterol 12: 4074-4077, 2006.

12. Nomura S, Sugano K, Kashiwabara H, Taniguchi T, Fukayama N, Fujita S, Akasu T, Moriya Y, Ohhigashi S, Kakizoe T and Sekiya T: Enhanced detection of deleterious and other germline mutations of hMSH2 and hMLH1 in Japanese hereditary nonpolyposis colorectal cancer kindreds. Biochem Biophys Res Commun 271: 120-129, 2000.

13. Fan Y, Liu X, Zhang H, Dai J, Zhang X, Zhu M, Gao X and Wang Y: Variations in exon 7 of the MSH2 gene and susceptibility to gastrointestinal cancer in a Chinese population. Cancer Genet Cytogenet 170: 121-128, 2006

14. Leung SY, Chan TL, Chung LP, Chan AS, Fan YW, Hung KN, Kwong WK, Ho JW and Yuen ST: Microsatellite instability and mutation of DNA mismatch repair genes in gliomas. Am J Pathol 153: 1181-1188, 1998.

15. Tang $\mathrm{R}$, Hsiung $\mathrm{C}$, Wang JY, Lai CH, Chien HT, Chiu LL, Liu CT, Chen HH, Wang HM, Chen SX, et al: Germ line MLH1 and MSH2 mutations in Taiwanese Lynch syndrome families: Characterization of a founder genomic mutation in the MLH1 gene. Clin Genet 75: 334-345, 2009.

16. Zhang Y, Liu X, Fan Y, Ding J, Xu A, Zhou X, Hu X, Zhu M, Zhang X, Li S, et al: Germline mutations and polymorphic variants in MMR, E-cadherin and MYH genes associated with familial gastric cancer in Jiangsu of China. Int J Cancer 119: 2592-2596, 2006.

17. Yuen ST, Chan TL, Ho JW, Chan AS, Chung LP, Lam PW, Tse CW, Wyllie AH and Leung SY: Germline, somatic and epigenetic events underlying mismatch repair deficiency in colorectal and HNPCC-related cancers. Oncogene 21: 7585-7592, 2002.

18. Jin HY, Yan HL, Song LH, Cui L, Ding YJ and Sun SH: Identification and functional analysis of a novel germ-line mutation in hMSH2 from a Chinese hereditary nonpolyposis colorectal cancer family. Acad J Sec Mil Med Univ 26: 888-891, 2005 (In Chinese).

19. Ng PC and Henikoff S: Predicting deleterious amino acid substitutions. Genome Res 11: 863-874, 2001.

20. Kumar P, Henikoff S and Ng PC: Predicting the effects of coding non-synonymous variants on protein function using the SIFT algorithm. Nat Protoc 4: 1073-1081, 2009.

21. Vander Molen J, Frisse LM, Fullerton SM, Qian Y, Del BosquePlata L, Hudson RR and Di Rienzo A: Population genetics of CAPN10 and GPR35: Implications for the evolution of type 2 diabetes variants. Am J Hum Genet 76: 548-560, 2005.

22. Nystrom-Lahti M, Perrera C, Raschle M, Panyushkina-Seiler E, Marra G, Curci A, Quaresima B, Costanzo F, D'Urso M, Venuta S and Jiricny J: Functional analysis of MLH1 mutations linked to hereditary nonpolyposis colon cancer. Genes Chromosomes Cancer 33: 160-167, 2002.

23. Clark AB, Cook ME, Tran HT, Gordenin DA, Resnick MA and Kunkel TA: Functional analysis of human MutSalpha and MutSbeta complexes in yeast. Nucleic Acids Res 27: 736-742, 1999.

24. Rodríguez-Negrete E, Bejarano ER and Castillo AG: Using the yeast two-hybrid system to identify protein-protein interactions. Methods Mol Biol 1072: 241-258, 2014

25. Zhu M, Fan YM, Zhu YB and Wang YP: Establishment of a hMSH2/hMSH6 protein interaction system and functional evaluation of hMSH2 gene missense mutations. Zhonghua Yi Xue Yi Chuan Xue Za Zhi 30: 559-564, 2013 (In Chinese).

26. Duraturo F, Liccardo R, Cavallo A, De Rosa M, Grosso M and Izzo P: Association of low-risk MSH3 and MSH2 variant alleles with Lynch syndrome: Probability of synergistic effects. Int J Cancer 129: 1643-1650, 2011.

This work is licensed under a Creative Commons Attribution-NonCommercial-NoDerivatives 4.0 International (CC BY-NC-ND 4.0) License. 